\title{
Hierarchical Virtual Experiment Design Based on 3ds MAX and VRP
}

\author{
Linlin Gao ${ }^{1, \text { a }}$, Zhikun Wang ${ }^{2}$ \\ ${ }^{1}$ Department of Quality Control Office, Yunnan Open University, Kunming Yunnan, China \\ ${ }^{2}$ College of Optoelectronics and Communication Engineering, Yunnan Open University, Kunming \\ Yunnan, China \\ aemail: 382082609@qq.com
}

Keywords: Level; Virtual Experiment; User Model; 3ds MAX; VRP-Bulider Editor

\begin{abstract}
Design and development of virtual experiments has become one of the focuses of the current research, thus the development of virtual experiment using 3ds MAX and VRP-Builder editor technology will also be the development trend of virtual experiment development. And 3ds MAX technology can realize the fidelity of the experimental model and the experimental scene, while VRP-Bulider editor technology can realize the high visualization of the scene, as well as the high interactivity and immersion of the experiment. At the same time, according to the user experience, domestic scholars divide the ergonomics into three Levels: perceptual Level, cognitive Level and physical Level. If the design of virtual experiment can combine the design philosophy of the three Levels' ergonomics, It can make the interface design and layout, interactive operation of virtual experiment be more accord with the physical and mental experience of learners, and be closer to the principles of nature.
\end{abstract}

\section{Introduction}

When designers design and develop virtual experiments, they often ignore the external form of technology that is, functional design, which leads to the poor usability of some virtual experiments, and if the experiment operation is too simple, it will easily cause the user's laziness, and it will be difficult to improve learners' ability and encourage them to challenge. Therefore, the experiment operation should be consistent with the current cognitive Level of users, and it should also have some certain difficulties to carry out the operation, making the operational program in the users' "zone of proximal development", and users should take some efforts to successfully operate the program, thus maximize their learning drive. Therefore, how to make the virtual experiment more in line with learners' cognitive characteristics and behavior habits, and to meet the hierarchical needs of learners are the most important questions in designing virtual experiment. The author attempts to introduce the hierarchical design theory of ergonomics into the researches on virtual experiment, and construct a hierarchical virtual experiment user model, so as to seek a feasible solution to optimize the quality of virtual experiment design.

\section{Overview of 3DS, MAX, and VRP-Bulider Editor Technologies}

\section{3ds MAX}

3D Studio Max, referred to as 3ds MAX, is a three-dimensional animation rendering and authoring software developed by Discreet Inc. (merged by Autodesk), based on the PC system. It grew out of the 3D Studio software based on the DOS operating system. In terms of the scope of application, it is widely used in advertising, film and television, industrial design, architectural design, three-dimensional animation, multimedia production, games, auxiliary teaching and engineering visualization, and so on. In this paper, 3ds MAX is mainly used to virtualize the experiment components and construct and exaggerate the 3D model of a screen. 


\section{VRP-Bulider Editor}

The VRP-Bulider editor is an independent virtual reality software developed by Vistandard Digital Technology Co., ltd. in Beijing, and because of the applicability and the simplicity of operation of the VRP-Bulider editor, which is very powerful, it has been widely used in urban planning, interior design, education and scientific research, the restoration of monuments, industrial simulation and military simulation and other fields. In this paper, VRP software is used as a development platform for virtual experiment system.

\section{Design of Hierarchical Virtual Experiment}

The hierarchical design idea aims at guiding the virtual experiment design with the system design idea, and improving the design quality and teaching effect of the experiment. The realization of hierarchical design of virtual experiment mainly includes the readability of symbols; functional visibility of an element; and the reversibility, fault tolerance, warning, acknowledgement, and assistance in interactive operations.

The three aspects of the hierarchical design of virtual experiment are interrelated, which also constitute a unified whole. The cognitive Level and physical Level design can enable learners to correctly and fluently operate the experiments, thus reducing errors and ensuring high availability of virtual experiments; the perceptual Level design is the highest realm of human-computer interaction with aesthetic meaning, thus ensuring the pleasure, freedom and sense of belonging of the learners in the process of experiencing. In view of the above, the author constructs a hierarchical virtual experiment user model(as shown in Figure 1).

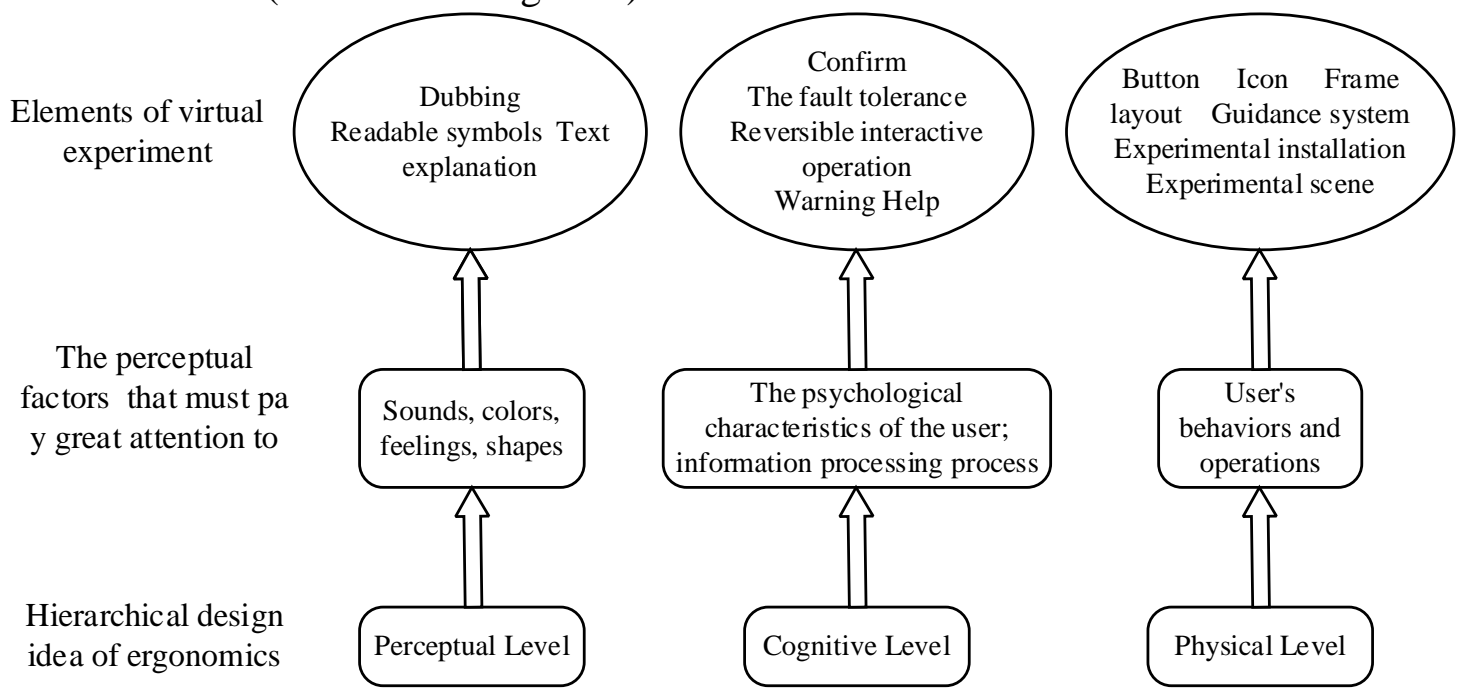

Fig.1. Hierarchical virtual experiment user model

\section{Design of the perceptual Level}

The highest Level of man-machine symbiosis design is the perceptual Level with aesthetic significance, and text explanation, dubbing as well as the readable symbols are the key elements of perceptual Level experience. In the learning process, learners can acquire knowledge more easily and efficiently with the help of feelings, sounds, colors, and so on, thus achieving personal satisfaction. Therefore, in the design of virtual experiment, emphasizes on perceptual Level design are equal to those on text explanation, dubbing as well as the readable symbols.

\section{Design of the cognitive Level}

- Reversibility of Interactive Operations

Navigation, interaction, and buttons in virtual experiments should be directed by learners' activities. When an activity is wrong, it is needed by learners to undo, return, and re-execution.

Reversible methods include: History panel—it can help learners define their own steps and locate where they need to return; go back to the previous step.

\section{- Fault Tolerance}

When designing virtual experiments, we should analyze the causes and types of errors. Designers 
should avoid interactive design that can lead to mis-operation, and give auxiliary hints to the operation steps with high error rate.

Virtual experiments should provide timely feedback according to the learners' operation to maintain the continuity of learning, so as to prevent disconnection or accumulating errors in the process of learning. The system should respond in a timely manner when learners are unable to operate normally by being prompted by the system, thus avoid an error of an operation which can fail the experiment. Error information that is fed back should not only inform the experimenter with the error, but also hint at the cause of the error. The language that describes the cause of the error should be easy to understand and specific to the content; the feedback should be friendly and varied, and give emotional experience to the experimenter through emotional words, sounds, images, or animations.

\section{- Warning}

There are two main ways of warning design: the first is symbol. Improper handling may produce certain dangerous signs, for example, if the operation is in the real experiment, it will cause serious damage if it is operated by mistake, learners should be warned about this type of problem; the second is the alarm. Simulate realistic alarms, sounds, and learners, certain behaviors that cause serious consequences will be limited to avoid danger.

\section{- Confirm}

Before the result of the learner's operation appears, a conversational confirmation should be given to avoid unexpected operation. A buffer phase should be given to the learner's important operations, for example, "Are you sure to leave without operation ? There are always two options, either yes or no.

\section{- Help}

The system can provide help to assist learners if they experience difficulties in the course of the experiment, such as disorientation and error. The help module provides online search, knowledge links, and online question answering, etc.

\section{Design of the Physical Level}

Icons, buttons, etc., can be designed to use symbols, pictures, etc. which are familiar to users, so that their functions can be seen, and with metaphor, the unfamiliar or difficult to understand information is expressed in simple and visual form, which is mainly showed in icon design, button design, guidance systems, frame layout, experimental installations, and experimental scene.

\section{Process Design}

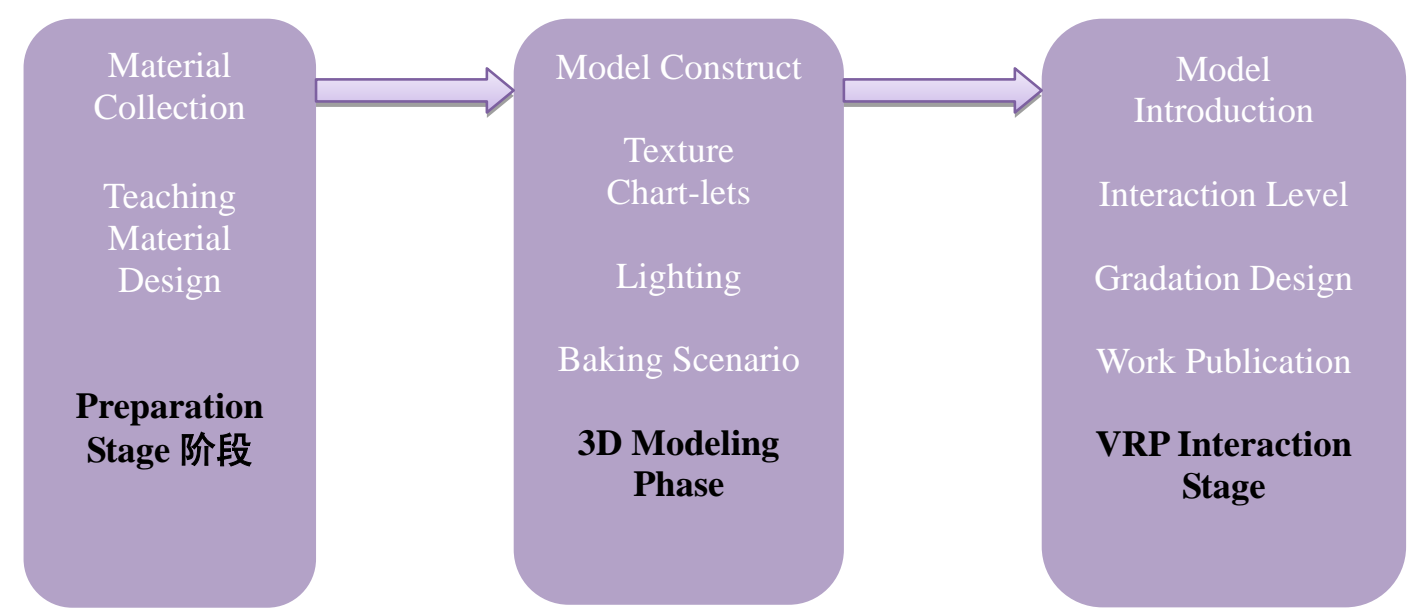

Fig.2. Flowchart of the virtual experiment system

According to the order of the system design, the implementation process of the virtual experiment system includes three steps: the preliminary stage, the 3D modeling stage and the interactive set-up stage, as shown in Figure 2.

\section{Collection of materials}

In the preliminary stage of system implementation, in addition to the preparation of "soft" 
material for instructional design (such as experimental scripts, dubbing, etc.), the collection of "hard" material produced by virtual scenes is also collected. The virtual experiment system is built on the basis of the physical laboratory. Therefore, it is necessary to collect the shapes, textures, textures, baking map photos, etc. of the laboratory.

\section{The establishment of three-dimensional model}

This paper uses the powerful and friendly interface of 3DS Max 2010 to construct the virtual experiment environment, which mainly includes the construction of the model of virtual laboratory, and virtual experiment instrument and equipment. After the model framework is created, add material and chart-lets to the model, and decorate the scene lights.

\section{Interactive design}

After completing the virtual experiment environment modeling in 3ds Max 2010, import it into the VRP editor through the installed VRP-for-Max plug-in, and you can preview when you import. After successfully importing the VRP-Bulider editor, the virtual experiment scene can be edited and adjusted, and interactive design can be implemented. The desktop virtual experiment system is realized in this paper, and learners interact with the system mainly through the mouse, keyboard, and interface buttons, and by setting the event of the model or button, the script command is triggered to interact with the objects in the scene, such as the translation of the model, zoom, rotate, and roaming in the scene, walking and so on.

\section{Release of Virtual Experiment System}

The release of VRP-Bulider editor virtual experiment system is relatively simple and flexible, files can be compiled into stand-alone exe files that can be executed independently, and can also be exported as Vrpie files for interactive browsing via the Internet.

\section{Case implementation}

Guided by the hierarchical virtual experiment user model constructed in this paper, and take virtual experiments in the Open University in Yunnan as cases, and expound the realization of hierarchical virtual experiment by using 3DS, Max, and VRP-Bulider editor techniques.

Realization of the Perceptual Level

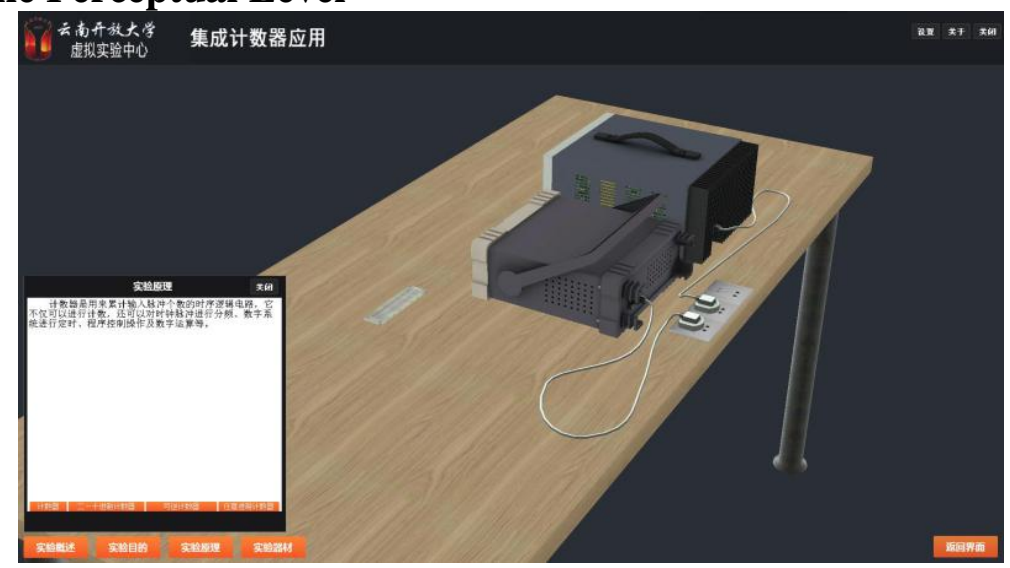

Fig.3. Experimental case of perceptual level: integrated counter application virtual experiment

The realization of perceptual Level focuses on two parts: Text interpretation and dubbing. As shown in Figure 3, in this experiment, the experimental principle is not only about the animation of the experimental principle, but also about the interpretation of the text and the explanation of the sound, which enables learners to acquire knowledge more easily and efficiently so as to achieve personal satisfaction. 


\section{Realization of the Cognitive Level}

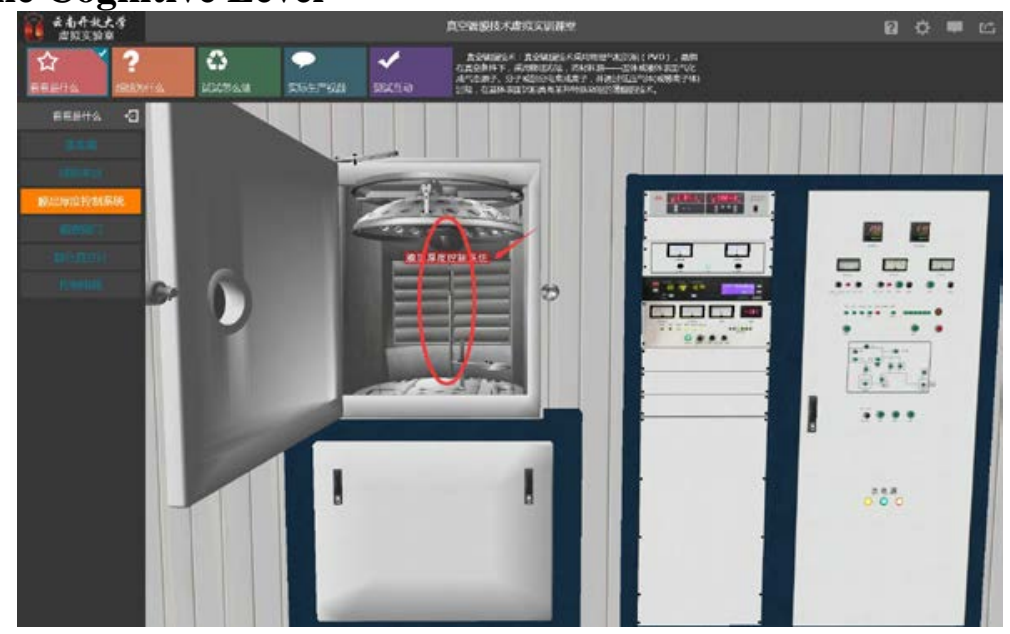

Fig.4. Experimental case of cognitive level: virtual experiment of vacuum coating

As shown in Figure 4, learners can independently explore the role and use of components, and when the operation goes wrong, the experiment will have an error message informing the experimenter of the error. At the same time, the system will pop up the undo, return, and re-execution buttons, meeting the needs of learners who wish to undo, return, and re execute. And it realizes the reversibility, fault tolerance and other functions of the cognitive level interaction design.

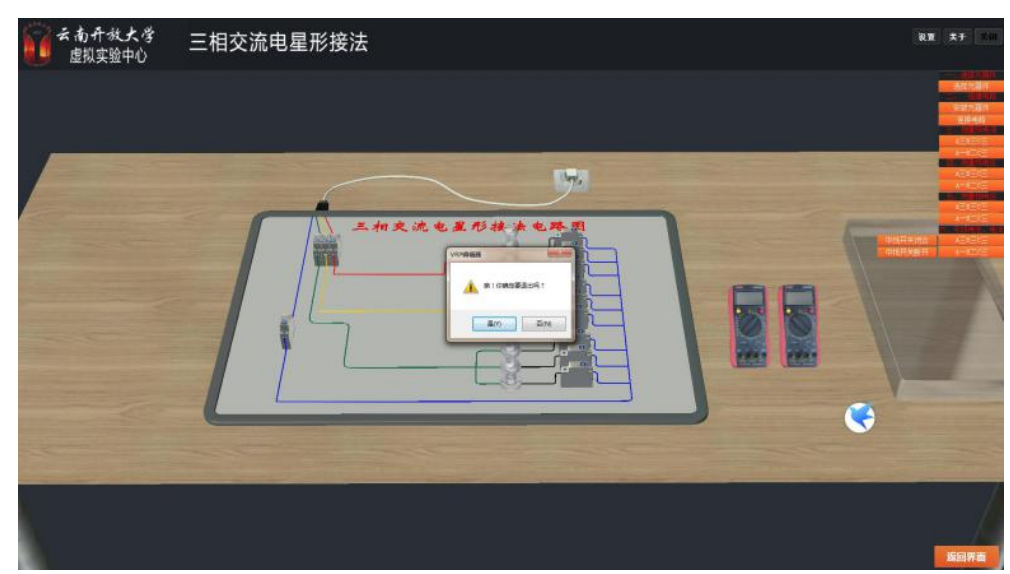

Fig.5. Cognitive level experimental case: virtual experiment of three - phase AC star connection

As shown in Figure 5, when the learner wants to withdraw from the study, a conversational confirmation will be given: "My dear friend, are you sure that you want to quit?", preventing learners from shutting down the experiment due to mis-operation. It realizes the function of the confirmation module at the cognitive level.

Realization of the Physical Level

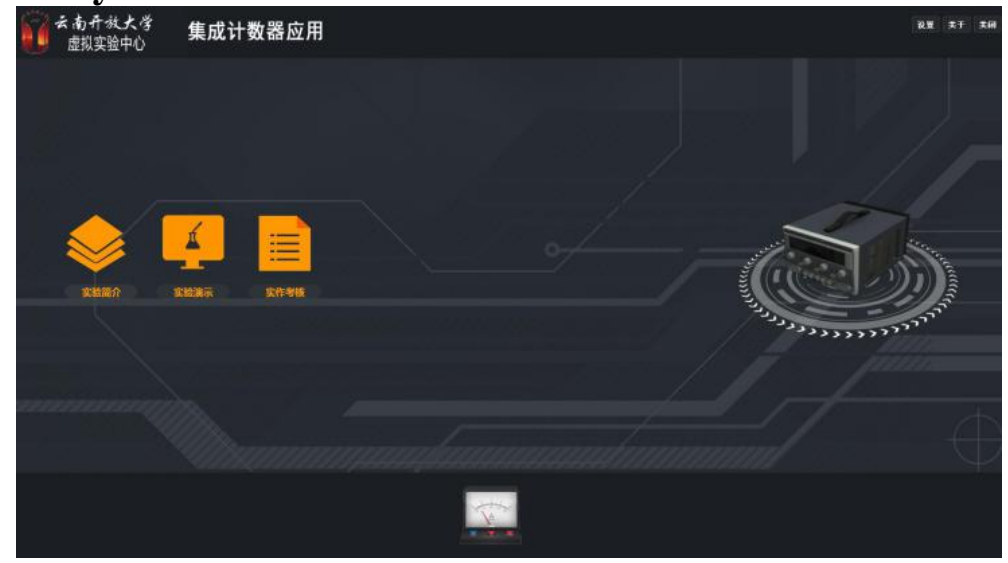

Fig.6. Physical level experimental case: integrated counter application virtual experiment 


\section{- Metaphor}

As shown in Figure 6, the design of the buttons of experiment demonstration, and examination all make use of the familiar symbols of the learners to make the function visible, and by using metaphor, the unfamiliar or difficult to understand information is expressed in simple and visual form. The visible aspects of physical hierarchical design functions are realized.

- Selective Focus

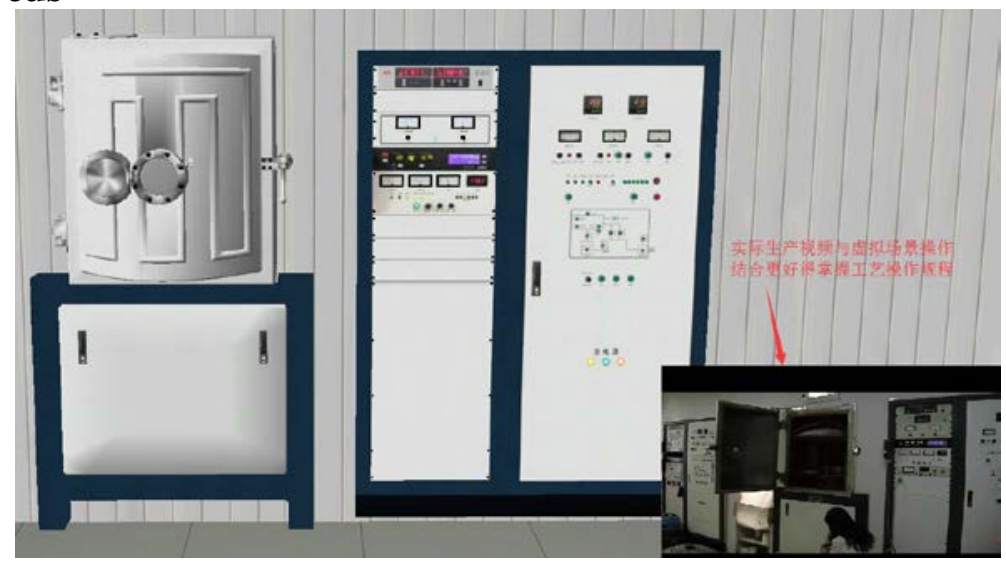

Fig.7. Selective focus experimental case: virtual experiment of vacuum coating

As shown in Figure 7, the project presentation introduces a method of combining actual production videos, which, on the one hand, is convenient for the learners to understand the concrete process of the actual production workshop and the processing of the instrument and equipment, on the other hand, is more convenient for learners to learn skills or pre job training with the hierarchical design of virtual and reality.

- Special Effects

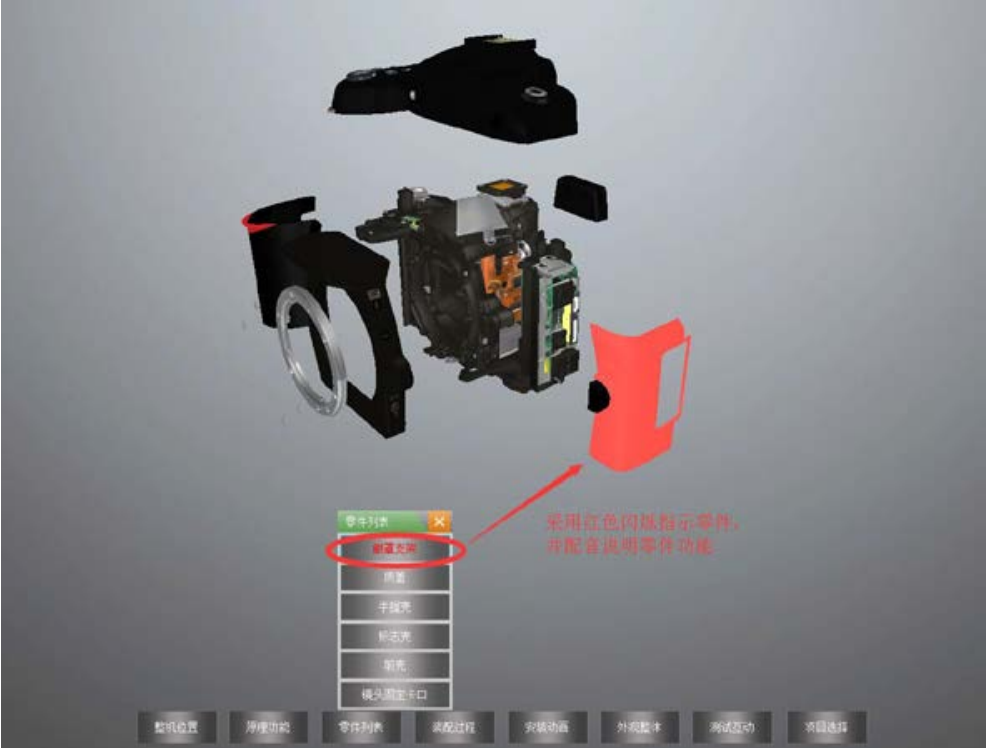

Fig.8. Experimental case of special effects: virtual assembly experiment of SLR camera

As shown in Figure 8, when learning the components of a structure, components are usually noted, or individually displayed, and in the development of this project, the special effects of dynamic mapping are adopted to facilitate the learners to clearly understand the results and position relations of the introduced parts.

The above three ways (metaphor, combination of virtual and reality, special effects) make the visibility of virtual experiment more clear, and ensures learners to operate more correctly and smoothly, thus reducing the occurrence of errors.

\section{Conclusion}

The development of virtual experiment using 3ds MAX and VRP-Builder editor technology will 
become the development trend of virtual experiment development, at the same time, the hierarchical design idea also provides a new way for improving the virtual experiment design. The connotation of the hierarchical design idea is coupled with the characteristics of virtual experiment elements, which provides a reference for designing virtual experiments that conform to learners' cognitive characteristics, behavioral habits and emotional experiences.

\section{References}

[1] Li Yujia. Research on the Rotation Design of Virtual Experiment[D]. Changchun: Jilin University, 2014

[2] Chen Li, Zhao Yiming. Design and Implementation of Research Virtual Experiment System Based on VRP. China Education Info，2014.07

[3] Chen Weidong. Research on Elastic Design of Virtual Experiment[D]. Changchun: Jilin University, 2015

[4] Su Linmeng, Kang Liuyi. Design of Virtual Experiment Based on 3DMax and VRP. Software Guide, 2014.10

[5] Kang Liuyi. Design and Development of Exploratory Virtual Experiment Based on 3DMax and VRP [D]. Northwest Normal University, 2014 